\title{
Event Anisotropy in High Energy Nucleus-Nucleus Collisions
}

\author{
H. Liu ${ }^{(1)}$, S. Panitkin ${ }^{(1)}$, and N. Xu ${ }^{(2)}$ \\ (1) Kent State University, Kent, Ohio 44242 \\ ${ }^{(2)}$ Lawrence Berkeley National Laboratory, Berkeley, California 94720
}

(June 29, 1998)

\begin{abstract}
The predictions of event anisotropy parameters from transport model RQMD are compared with the recent experimental measurements for $158 \mathrm{~A} \mathrm{GeV}$ $\mathrm{Pb}+\mathrm{Pb}$ collisions. Using the same model, we study the time evolution of event anisotropy at $2 A \mathrm{GeV}$ and $158 \mathrm{~A} \mathrm{GeV}$ for several colliding systems. For the first time, both momentum and configuration space information are studied using the Fourier analysis of the azimuthal angular distribution. We find that, in the model, the initial geometry of the collision plays a dominant role in determining the anisotropy parameters.
\end{abstract}

Typeset using REVTEX 
Event anisotropy, often called flow, has been observed in heavy ion collisions at every laboratory energy [1 [1]. It is believed that the information about the equation of state (EOS) can be obtained from the study of flow [10]. Recently, several authors argue that the event shape with respect to the reaction plane may carry the information about pressure created at the early stage of the collision [11] [15].

Theoretically, in terms of hydrodynamics, the connection between the EOS and flow is well defined and has been studied extensively for many years. This is especially the case for collisions at beam energy below $15 \mathrm{~A} \mathrm{GeV}$. (For the latest review of the subject, see Ref. [9]16].) However, it is still not clear whether the experimentally observed event anisotropy is of a dynamic origin (pressure-driven hydroexpansion) or due to shadowing of cold nuclear matter, passing time etc. If the initial geometry of the system plays an important role, the information about the collision dynamics may be obscured. In any case, it seems to be necessary to understand the interplay between these two competing effects. For this purpose, we use the transport model RQMD(v2.3 cascade mode) [17] to study the event anisotropy as a function of the colliding system and beam energy. We also investigate the collision time dependence of the event shapes.

In RQMD, the reaction plane is defined by the impact parameter ( $x$ direction) and the projectile momentum ( $z$ direction). Particle azimuthal distribution with respect to the reaction plane at a given rapidity window can be deconvoluted by the Fourier expansion [24,

$$
\frac{d N}{d \phi} \approx v_{0}\left(1+2 v_{1} \cos (\phi)+2 v_{2} \cos (2 \phi)\right)
$$

where the first and second Fourier coefficients, $v_{1}$ and $v_{2}$, are connected to the directed flow and elliptic flow, respectively [24]. The coefficient $v_{0}$ is a normalization constant and $\phi$ is defined as the azimuthal angle difference between the particle and the reaction plane. In our case, the reaction plane angle is explicitly set to zero. Hence $\phi$ angle in momentum space is $\phi=\tan ^{-1}\left(p_{y} / p_{x}\right)$, and in configuration space $\phi=\tan ^{-1}(y / x)$. At a given rapidity, the coefficients are: 


$$
v_{1}=\langle\cos (\phi)\rangle, \quad v_{2}=\langle\cos (2 \phi)\rangle
$$

In the following, we will first compare the recent NA49 measurements of Fourier moments with RQMD calculations. In section II and III, the colliding system dependence and time evolution of anisotropy parameters $v_{1}$ and $v_{2}$ will be discussed. Finally, a brief summary will be presented in section IV.

\section{COMPARISON OF DATA WITH MODEL PREDICTIONS FOR 158 A GEV PB+PB COLLISIONS}

The experimentally measured $\left(v_{1}, v_{2}\right)$ parameters for proton and pion as a function of rapidity are shown as filled circles in Figure 1. These data are extracted from the $158 \mathrm{~A} \mathrm{GeV}$ $\mathrm{Pb}+\mathrm{Pb}$ collisions by the NA49 collaboration [6.7]. More details about methods involved in the experimental determination of these parameters can be found in the recent paper [19]. The model calculations (open circles) are done within similar impact parameter range and acceptance $\left(p_{t}, y\right)$ cuts. As one can see, the model calculations are in reasonable agreement with the data for both protons and pions. The sign of the first Fourier moment $v_{1}$ for pions is opposite to that of protons for both data and model calculations indicating the nuclear shadowing effects in the collision. An interesting feature in Figure 1 is that there seems to be two slopes for the function $v_{1}(y)$ : at $|y| \leq 1.5$, the distribution is relatively flatter compared to the region $|y|>1.5$. This implies that the physics around the mid-rapidity region is different from that near the projectile (target) rapidity region. One possible interpretation is that near the beam rapidity region, the number of rescatterings is small, therefore the initial geometry leads to a large value of $v_{1}$. On the contrary, a large number of rescatterings washes out the initial memory and leaves small anisotropy around the mid-rapidity.

As predicted in Ref. [20], the second Fourier moment $v_{2}$ is found to be positive for both nucleons and pions at this beam energy. This implies an enhancement of in-plane emission from the particle source. More discussions on this point will be presented later in this paper.

Since the model predictions are in reasonable agreement with the experimental data, it 
may be instructive to study system size dependence and the space-time evolution of the anisotropy parameters within the model.

\section{II. $V_{1}$ FOR DIFFERENT COLLISION SYSTEMS}

In Figure 2, the model predicted $v_{1}$ for nucleons and pions as a function of rapidity are shown for $2 A \mathrm{GeV} \mathrm{S}+\mathrm{S}(3 \leq b \leq 5 \mathrm{fm}), 2 A \mathrm{GeV} \mathrm{Ru}+\mathrm{Ru} \Psi(4 \leq b \leq 6 \mathrm{fm}), 2 A \mathrm{GeV} \mathrm{Pb}+\mathrm{Pb}$ $(5 \leq b \leq 8 \mathrm{fm}), 158 A \mathrm{GeV} \mathrm{S}+\mathrm{S}(3 \leq b \leq 5 \mathrm{fm})$ and $158 A \mathrm{GeV} \mathrm{Pb}+\mathrm{Pb}(5 \leq b \leq 8 \mathrm{fm})$ collisions. The impact parameter range is chosen such that the maximum value of $v_{1}$ is achieved for any given colliding system.

It is known from the hydrodynamic calculations that the pressure created at the early stage will lead to sizable values of $v_{1}$ and $v_{2}$, and the pressure is different for different bombarding energies [21]. However, from Figure 2 and Table [], one notices that the maximum values of $v_{1}$ for nucleons are independent of the beam energy for a given colliding system. For a fixed beam energy, the maximum value of $v_{1}$ depends on the size of the colliding nuclei. The larger the size, the higher the maximum value of $v_{1}$. This may suggest that the initial geometry, i.e. the size of the colliding nuclei and impact parameter, determines the strength of $v_{1}$ near the beam (target) rapidity region, providing the elastic scattering amplitude does not vary much within energy range discussed here. Similar trends are also seen for pions even though the $v_{1}$ for pions has the opposite sign to that of nucleons due to the shadowing effect.

The other interesting feature is that the slope of the $v_{1}$ curve around the mid-rapidity region depends on beam energy for both nucleons and pions. The higher the beam energy, the smaller the slope']. The value of $v_{2}$, on the other hand, is maximized around mid-rapidity. It was suggested that the second Fourier moment may carry the information about the early

\footnotetext{
${ }^{1}$ The exact beam energy for the $\mathrm{Ru}+\mathrm{Ru}$ collisions is $1.69 \mathrm{~A} \mathrm{GeV}$.

${ }^{2}$ This is also true for $v_{1}$ as a function of normalized rapidity.
} 
stage of the collision [11,15]. The sensitivity of the $v_{2}$ signal to the collision dynamics will be discussed in the next section.

\section{TIME EVOLUTION OF $\left(V_{1}, V_{2}\right)$}

Now let us explore $\left(v_{1}, v_{2}\right)$ of nucleons and pions as a function of freeze-out time for two colliding systems: $2 A \mathrm{GeV} \mathrm{Au}+\mathrm{Au}$ and $158 A \mathrm{GeV} \mathrm{Pb}+\mathrm{Pb}$ collisions. In a discussion of the time evolution, a brief remark concerning the nuclear passing time is necessary. Nuclear passing time is the time needed for two incoming nuclei to pass each other and defined as $t_{p}=2 r /(\gamma \beta)$ where $r$ is the nuclear radius. Passing time sets the scale on which the effects of possible nuclear shadowing, e.g. the interaction of the participating nucleons and produced particles with the spectator matter, are significant. Obviously, the passing time depends on the beam energy and on the size of colliding nuclei. The nuclear passing time $t_{p}$, in the nucleon-nucleon center-of-mass frame, for $2 A \mathrm{GeV} \mathrm{Au}+\mathrm{Au}$ and $158 A \mathrm{GeV} \mathrm{Pb}+\mathrm{Pb}$ collisions are listed in Table [1]. Also listed are the nucleon mean freeze-out time $t_{m}$ from the RQMD calculations. The ratio of $t_{m} / t_{p}$ for the low energy collisions is much smaller than that at the high energy, indicating that the nuclear shadowing is relatively more important for lower energy collisions.

\section{A. $v_{1}, v_{2}$ (momentum space)}

The calculated $\left(v_{1}, v_{2}\right)$ values, determined in the momentum space, as a function of rapidity in different time windows are shown in Figures 3 and 4 for nucleons and pions, respectively. In these figures, the open circles are for the lower energy $(2 A \mathrm{GeV})$ and the filled circles for the higher energy $(158 \mathrm{~A} \mathrm{GeV})$ collisions. Similar distributions calculated at the corresponding passing time $(0 \leq t \leq 12 \mathrm{fm} / \mathrm{c}$ for $2 A \mathrm{GeV}$ and $0 \leq t \leq 2 \mathrm{fm} / \mathrm{c}$ for $158 A$ GeV collisions) are shown in Figure 5.

For nucleons, as one can see in Figure 3 (left column lower 4 plots), the strength of $v_{1}$ is gradually built up as a function of collision time. At the earlier time, $t \leq 10 \mathrm{fm} / \mathrm{c}$, a 
well defined negative slope (around mid-rapidity) is seen for the low energy collisions while at the high energy the distribution is relatively flat. This flatness is due to the averaging over the $10 \mathrm{fm} / \mathrm{c}$ time period which is much longer than the passing time for the $158 \mathrm{~A} \mathrm{GeV}$ collisions (see Table [I]). In fact, the negative slope is also present at that energy for $t \leq t_{p}$ as shown in Figure 5. This again suggests that the shadowing of the cold nuclear matter is responsible for the observed negative slope at the early stage of the collision. At $t>10$ $\mathrm{fm} / \mathrm{c}$, the maximum value of $v_{1}$ has been well established for the lower energy collisions. For the higher energy collisions, the fully established $v_{1}$ only appears after $t>30 \mathrm{fm} / \mathrm{c}$.

The time dependence of $v_{1}$ for pions shows a different trend (Figure 4 left column). The maximum values of $v_{1}$ are achieved at the early stage of the collision. As time goes on, the distributions of $v_{1}$ become flatter. The fact that the $v_{1}$ distributions for pions always have a negative slope through the whole collision history suggests the importance of shadowing effect. However, the shadowing matter is different for pions at different collision stages. At early time, $t \leq t_{p}$, pions (as well as the participating nucleons) are shadowed by the cold spectators. Later, after the spectator matter leaves the collision zone, pions are shadowed by the participant nucleons. This may be the underlying mechanism that leads to the different behavior of $v_{1}$ for nucleons and pions.

The second Fourier coefficient $v_{2}$ describes an elliptical shape of the azimuthal distribution. A positive sign of $v_{2}$ means that the longer axis of the ellipse is oriented in the reaction plane while a negative sign of $v_{2}$ indicates the longer axis is in the direction perpendicular to the reaction plane. This latter effect was called "squeeze-out" at Bevalac energies [22]. In Figure 3 and Figure 1 (right column), the $v_{2}$ distributions are shown as a function of rapidity in different time intervals for nucleons and pions, respectively. It can be seen that the time averaged values of $v_{2}$ for nucleons and pions at both beam energies are positive, implying that particles preferentially freeze-out in the reaction plane.

However, at the time scale comparable with nuclear passing time, nucleons and pions demonstrate an out-of-plane emission pattern ("squeeze-out") for $2 A \mathrm{GeV}$ collisions (Figure 5 right columns) which is consistent with the cold nuclear matter shadowing scenario. For 
high energy collisions, the "squeeze-out" effect is absent for both pions and nucleons. This may be explained by a much shorter nuclear passing time and higher Lorentz contraction of the colliding nuclei. According to the RQMD calculations, at about the corresponding passing time, the average number of collisions suffered by the mid-rapidity nucleons is 3 and 5 for $158 A \mathrm{GeV}$ and $2 A \mathrm{GeV}$ collisions, respectively. In the high energy collisions the rapidity span is about 6 units. Hence, three collisions are just about enough to move a nucleon from projectile rapidity to mid-rapidity. At this energy, neither time nor number of rescatterings is sufficient to develop an out-of-plane emission.

\section{B. $v_{1}, v_{2}$ (configuration space)}

Up to now, we have discussed the anisotropy parameters $\left(v_{1}, v_{2}\right)$ in the momentum space. It is interesting to look at these parameters in coordinate space in order to understand the space-time evolution of the collisions within the model. To the best of our knowledge such studies have not been performed before. The information about source anisotropy may be obtained experimentally by studying two-particle correlation functions [23 26].

In general, the $v_{1}$ parameters in the configuration space for both nucleons and pions (see Figure 6 and 7 left columns) behave differently from those in the momentum space: (1) The shapes of the $v_{1}$ distributions are different and the maximum values of $v_{1}$ (around the beam and target rapidities) in the configuration space are much larger than those in the momentum space; (2) The $v_{1}$ of pions has the same sign to that of nucleons; (3) The $v_{1}$ becomes stronger as a function of time, especially for pions. Furthermore, similar to what have been observed in the momentum space, the maximum values of $v_{1}$ are the same for the two collisions at different beam energies. This implies that the initial collision geometry plays an important role here.

For the non-zero impact parameter heavy-ion collisions, the transverse shape of the initial collision zone is an ellipse with its longer axis oriented perpendicular to the reaction plane $\left(v_{2}<0\right)$. Such an event shape is indeed seen for both collision energies in Figure 6 
(right column) for the time interval $t \leq 10 \mathrm{fm} / \mathrm{c}$. As time goes on, rescattering occurs among particles which leads to a more spherical shape of the reaction zone, $v_{2} \rightarrow 0$. Note one would expect a large positive $v_{2}$ at later times in the case of pressure driven hydroexpansion. For the time integrated distributions, some remnants of the initial shape still can be seen for the $2 A \mathrm{GeV}$ collisions while for the higher energy collision the source appears to be isotropic.

\section{SUMMARY}

Based on the RQMD(v2.3) model calculations, we studied the anisotropy parameters $\left(v_{1}, v_{2}\right)$ as a function of time for different energies and different colliding systems. From a comparison between the model calculations and the recent experiment measurement at the beam energy of $158 \mathrm{~A} \mathrm{GeV}$, we find that the model provides a reasonable description of the observed event anisotropy. From the study of energy dependence, we find that the combination of collision geometry and nuclear passing time plays a dominant role in determining the behavior of the $v_{1}$ and $v_{2}$ parameters. Our studies show that the anisotropy parameters extracted in the momentum space are different from the ones extracted in the configuration space. As a function of collision time, some rather dramatic changes are found for the event anisotropy parameters in both momentum and configuration spaces. At $t \leq t_{p}$, the pattern of the distributions is strongly influenced by the spectators. At later times, a prominent in-plane emission is evident.

Acknowledgments We wish to express our gratitude to Drs. J. Kapusta, D. Keane, V. Koch, A. Poskanzer, G. Rai, H.G. Ritter, H. Sorge, H. Stöcker, and S. Voloshin for many helpful discussions. We especially thank Dr. H. Sorge for providing the RQMD code. This research used resources of the National Energy Research Scientific Computing Center. This work has been supported by the U.S. Department of Energy under Contract No. DE-AC0376SF00098 and National Science Foundation. 


\section{REFERENCES}

[1] M. Partlan et al., EOS Collaboration, Phys. Rev. Lett. 75, 2100(1995).

[2] P. Crochet, FOPI collaboration: nucl-ex/9709004, to appear in Nucl. Phys. A

[3] H. Liu, for the E895 collaboration, Quark Matter '97, Tsukuba, Japan, Dec. 1-5, 1997.

[4] J. Barrette et al., E877 Collaboration, Phys. Rev. C55, 1420(1997).

[5] S.Voloshin, for the E814/E877 collaboration, Quark Matter '97, Tsukuba, Japan, Dec. 1-5, 1997.

[6] A. Poskanzer, for the NA49 collaboration, Quark Matter '97, Tsukuba, Japan, Dec. 1-5, 1997.

[7] H. Appelshauser et al., NA49 Collaboration, Phys. Rev. Lett. in print.

[8] S. Nishimura, for the WA98 collaboration, Quark Matter '97, Tsukuba, Japan, Dec. 1-5, 1997.

[9] W. Reisdorf and H.G. Ritter, Annu. Rev. Nucl. Part. Sci. 47, 663(1997).

[10] L.P. Csernai, "Introduction to Relativistic Heavy Ion Collisions", John Wiley \& Sons, 1994.

[11] H. Sorge, Phys. Rev. Lett. 78, 2309(1997).

[12] H. Sorge, 'Advances in Nuclear Dynamics 3', Page 1, ed. Bauer and Mignerey, Plenum Press, New York, 1997.

[13] D.H. Rischke, Nucl. Phys. A610 88c(1996).

[14] M. Gyulassy, D.H. Rischke and B. Zhang, Nucl. Phys. A613, 397(1997).

[15] P. Danielewicz et al., Los Alamos Preprint nucl-th/9803047 26 Mar 1998.

[16] P. Danielewicz, Phys. Rev. C51, 716(1995). 
[17] H. Sorge, Phys. Rev. C52 3291(1995).

[18] S. Voloshin and Y. Zhang, Z. Phys. C70, 665(1996).

[19] A. Poskanzer and S. Voloshin, Phys. Rev. C, in print; Los Alamos Preprint nuclex/9805001.

[20] J.-Y. Ollitrault, Phys. Rev. D46, 229(1992).

[21] B. Blättel, V. Koch, and U. Mosel, Rep. Prog. Phys. 56, 1(1993).

[22] H.H. Gutbrod et al., Phys. Rev. C42, 640(1990).

[23] S. Voloshin, R. Lednicky, S. Panitkin, and N. Xu, Phys. Rev. Lett., 79, 4766(1998).

[24] S. Voloshin and W. Cleland, Phys. Rev. C54, 3112(1996).

[25] U.A. Wiedemann, Phys. Rev. C57, 266(1998).

[26] S. Panitkin and N. Xu, in preparation. 


\section{TABLES}

\begin{tabular}{|c||c|c|c|c|}
\hline \hline Colliding System & $A$ & $\mathrm{r}(\mathrm{fm})$ & $\mathrm{y}\left(v_{1}^{\max }\right)$ & $v_{1}^{\max }$ \\
\hline $2 A \mathrm{GeV} \mathrm{S}+\mathrm{S}$ & 32 & 3.8 & 0.91 & $0.171 \pm 0.007$ \\
\hline $2 A \mathrm{GeV} \mathrm{Ru}+\mathrm{Ru}$ & 96 & 5.5 & 0.91 & $0.186 \pm 0.003$ \\
\hline $2 \mathrm{~A} \mathrm{GeV} \mathrm{Pb}+\mathrm{Pb}$ & 208 & 7.1 & 0.91 & $0.225 \pm 0.009$ \\
\hline \hline $158 \mathrm{~A} \mathrm{GeV} \mathrm{S}+\mathrm{S}$ & 32 & 3.8 & 2.92 & $0.166 \pm 0.008$ \\
\hline $158 \mathrm{~A} \mathrm{GeV} \mathrm{Pb+Pb}$ & 208 & 7.1 & 2.92 & $0.225 \pm 0.009$ \\
\hline \hline
\end{tabular}

TABLE I. Nucleon $v_{1}$ parameters for symmetric $A-A$ collisions, the atomic number $A$, the radius $\left(r=1.2 \cdot A^{1 / 3}\right)$ of the beam(target) nucleus and the rapidity corresponding to the maximum value of $v_{1}$ are shown.

\begin{tabular}{|c||c|c|c|c|c|}
\hline \hline Energy & $\beta(c)$ & $\gamma$ & $y$ & $t_{p}(\mathrm{fm} / \mathrm{c})$ & $t_{m}(\mathrm{fm} / \mathrm{c})$ \\
\hline $2 \mathrm{GeV}$ & 0.718 & 1.438 & 0.904 & 13.52 & 20.63 \\
\hline $158 \mathrm{GeV}$ & 0.994 & 9.231 & 2.913 & 1.55 & 33.89 \\
\hline \hline
\end{tabular}

TABLE II. Velocity $\beta$ (in the unit of speed of light), $\gamma$, rapidity $y$ and passing time $t_{p}$ are

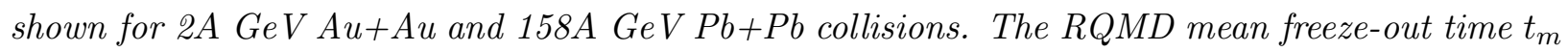
for nucleons are also included. All quantities are evaluated in the nucleon-nucleon center-of-mass frame. 


\section{FIGURES}

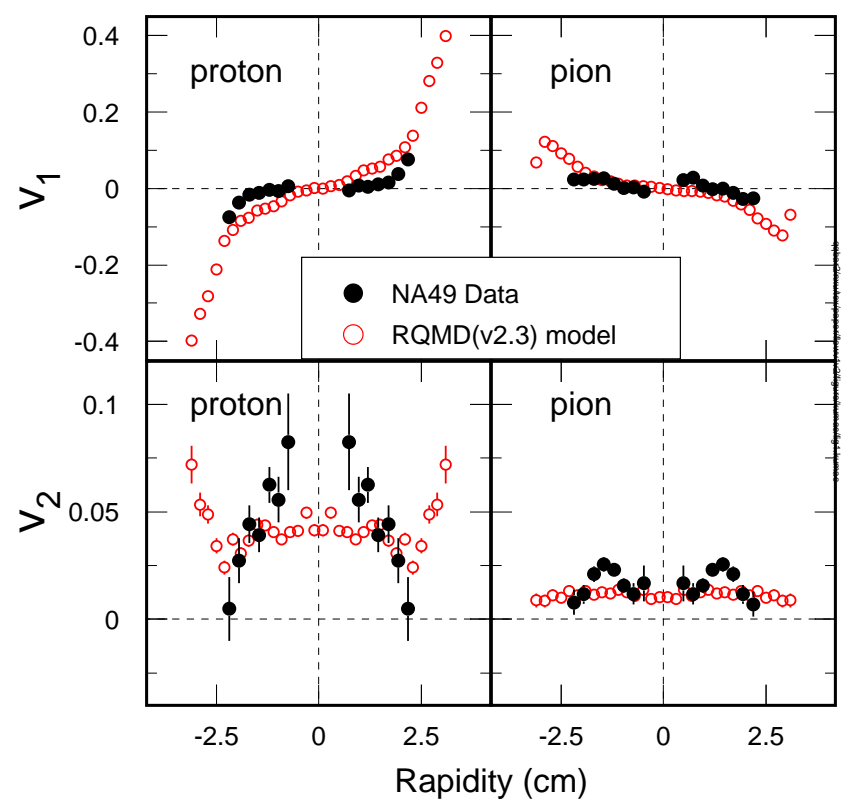

FIG. 1. $v_{1}, v_{2}$ for proton and pion as a function of rapidity (filled circles - experimental results, open circles - model calculations) from $158 A \mathrm{GeV} \mathrm{Pb}+\mathrm{Pb}$ collisions. 


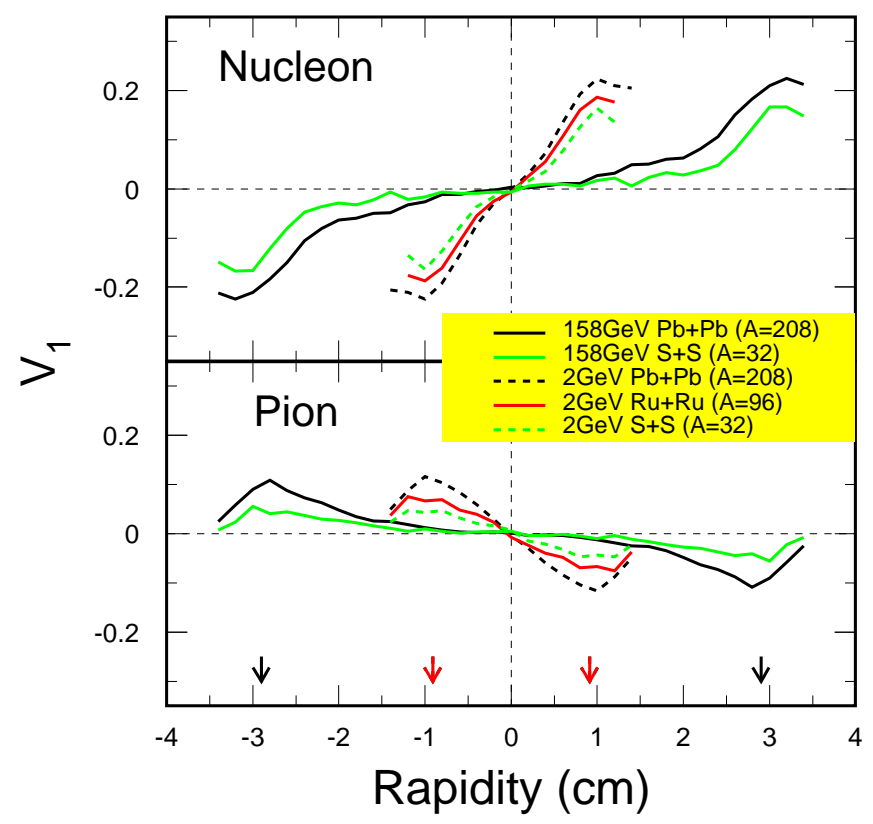

FIG. 2. $\quad v_{1}$ as a function of rapidity $y$, in center-of-mass frame, for nucleons and pions for different colliding systems. The arrows indicate the beam and target rapidities. 


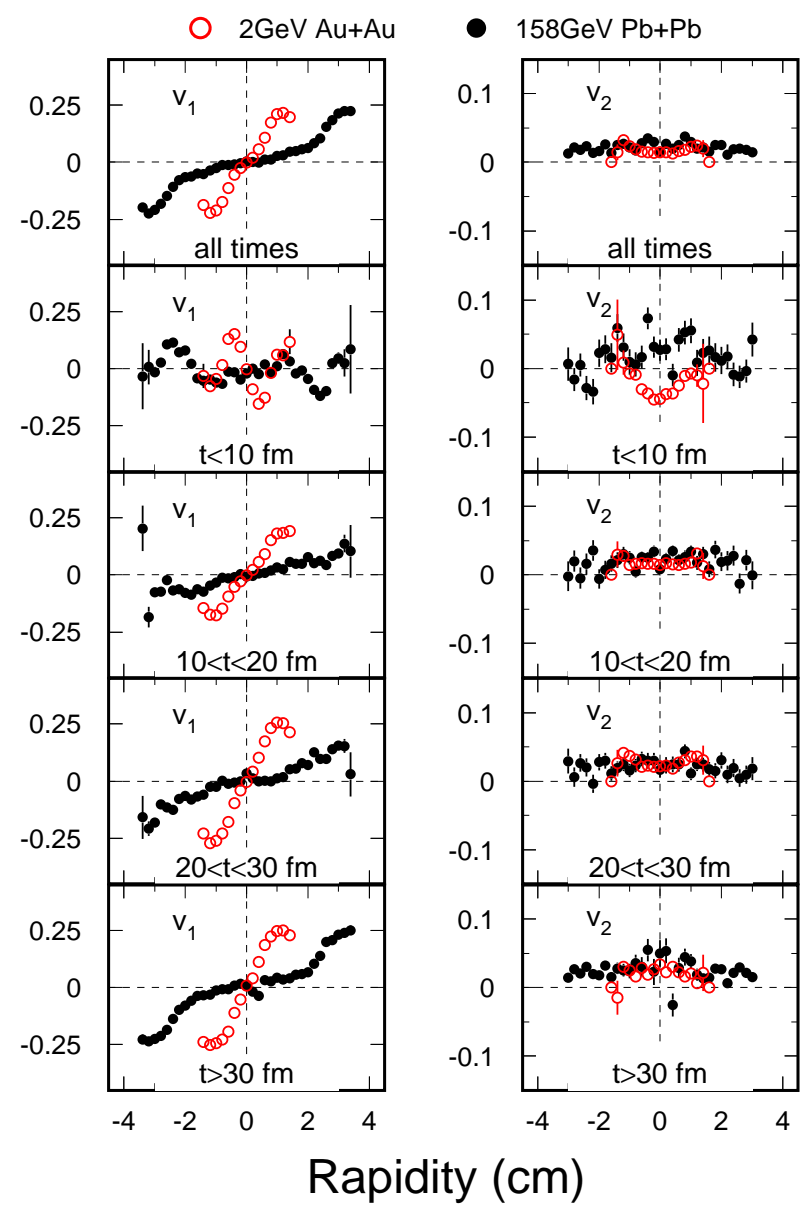

FIG. 3. The time evolution of $v_{1}$ and $v_{2}$ for nucleons in the momentum space as a function of rapidity in the center-of-mass frame for $2 A \mathrm{GeV} \mathrm{Au}+\mathrm{Au}$ collisions (open circles) and $158 \mathrm{~A} \mathrm{GeV}$ $\mathrm{Pb}+\mathrm{Pb}$ collisions (filled circles). 


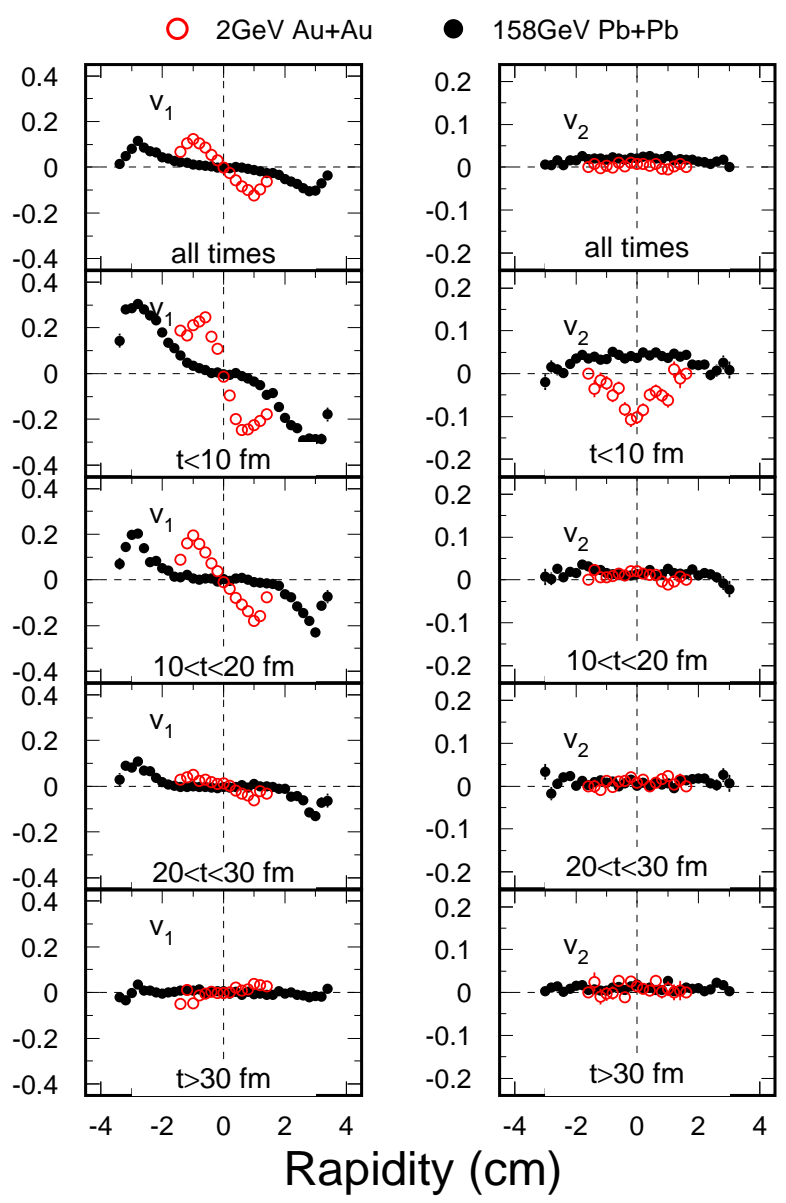

FIG. 4. The time evolution of $v_{1}$ and $v_{2}$ for pions in the momentum space as a function of rapidity in the center-of-mass frame for $2 A \mathrm{GeV} \mathrm{Au+Au}$ collisions (open circles) and $158 A \mathrm{GeV}$ $\mathrm{Pb}+\mathrm{Pb}$ collisions (filled circles). 


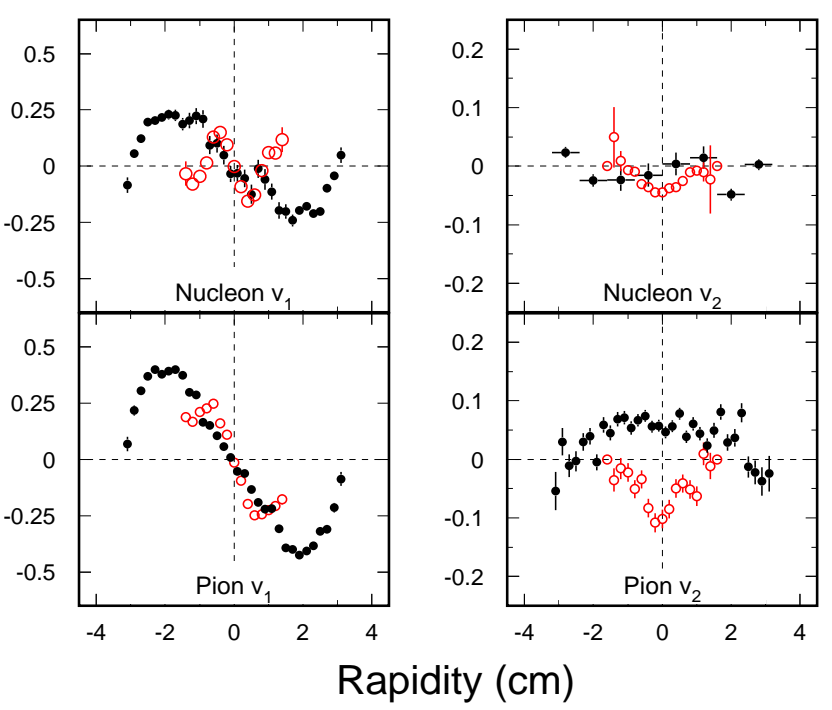

FIG. 5. Nucleon and pion anisotropic parameters $v_{1}, v_{2}$ as a function of rapidity, around the nuclear passing time, for $2 A \mathrm{GeV} \mathrm{Au}+\mathrm{Au}$ (open circles) and $158 \mathrm{~A} \mathrm{GeV} \mathrm{Pb}+\mathrm{Pb}$ (filled circles) collisions. 


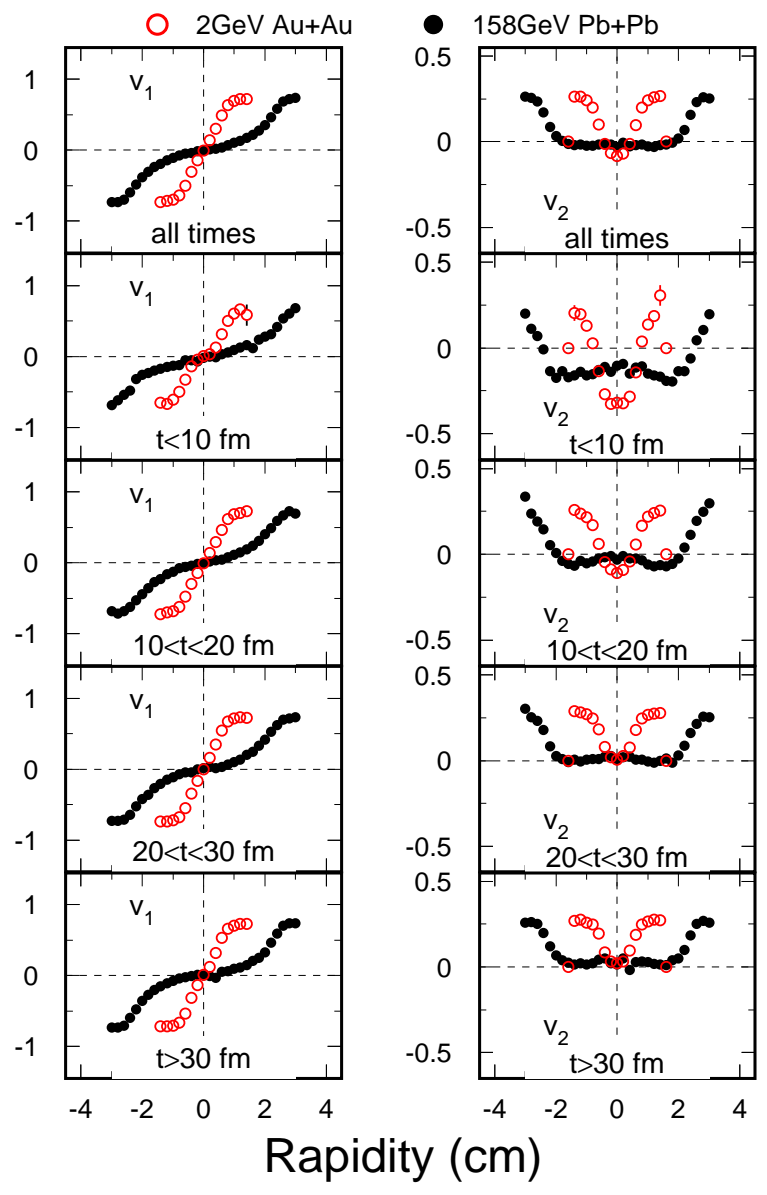

FIG. 6. The time evolution of $v_{1}$ and $v_{2}$ for nucleons in the configuration space as a function of rapidity in the center-of-mass frame for $2 A \mathrm{GeV} \mathrm{Au}+\mathrm{Au}$ collisions (open circles) and $158 \mathrm{~A} \mathrm{GeV}$ $\mathrm{Pb}+\mathrm{Pb}$ collisions (filled circles). 


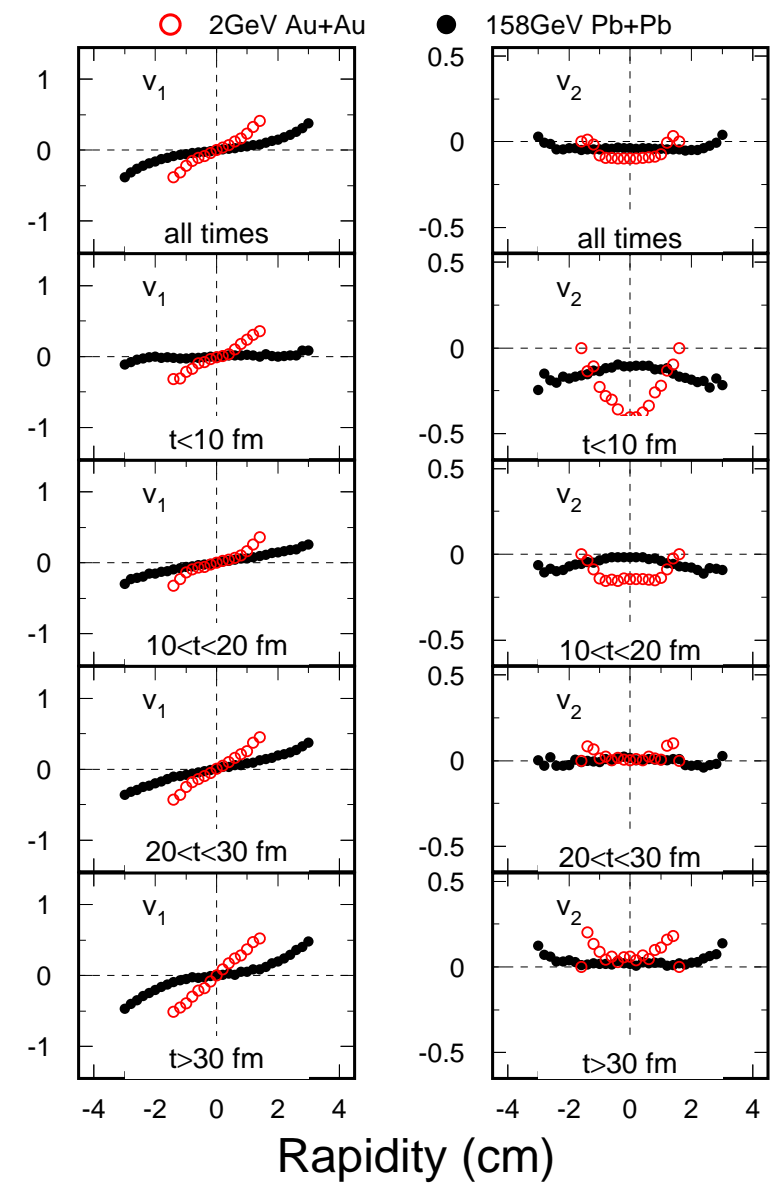

FIG. 7. The time evolution of $v_{1}$ and $v_{2}$ for pions in the configuration space as a function of rapidity in the center-of-mass frame for $2 A \mathrm{GeV} \mathrm{Au}+\mathrm{Au}$ collisions (open circles) and $158 \mathrm{~A} \mathrm{GeV}$ $\mathrm{Pb}+\mathrm{Pb}$ collisions (filled circles). 


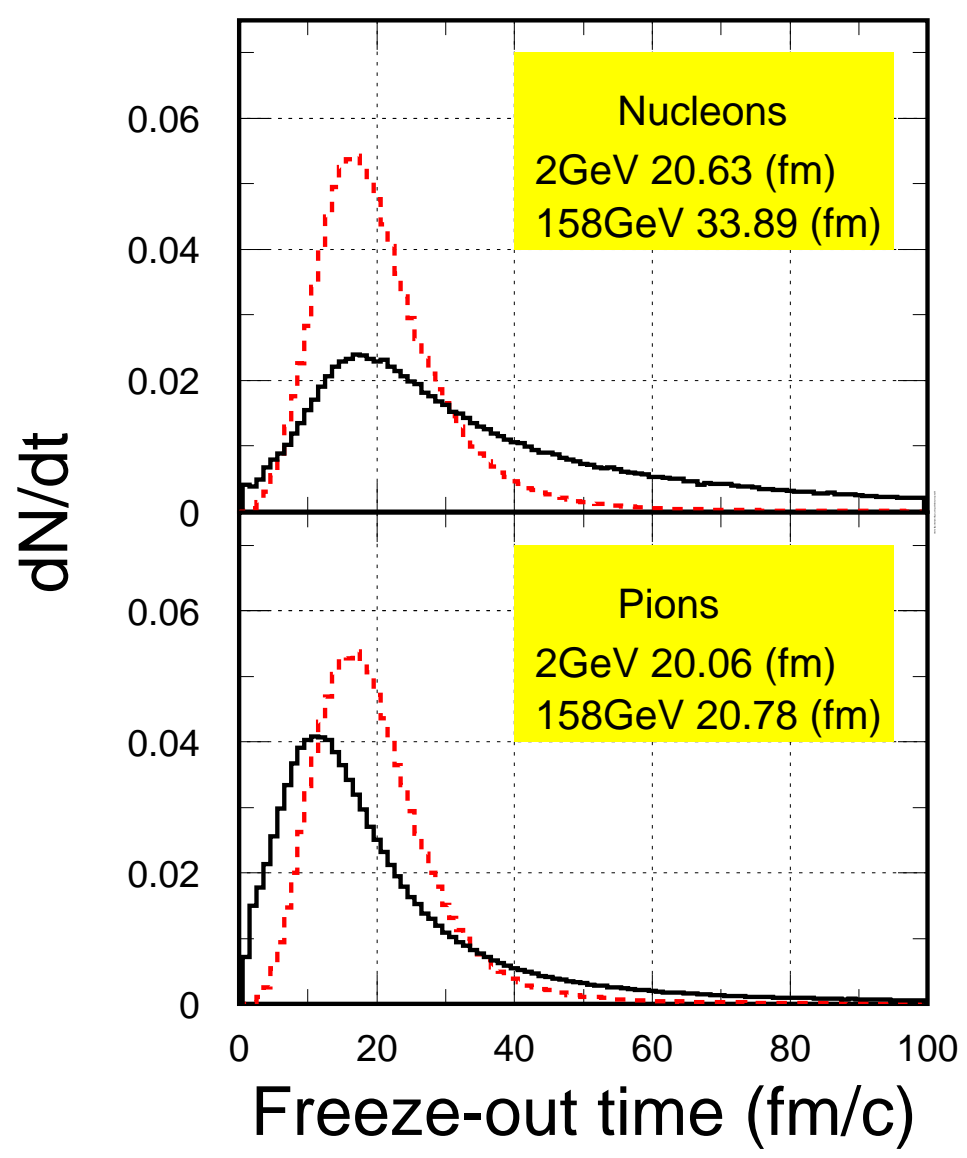

Fig.2 


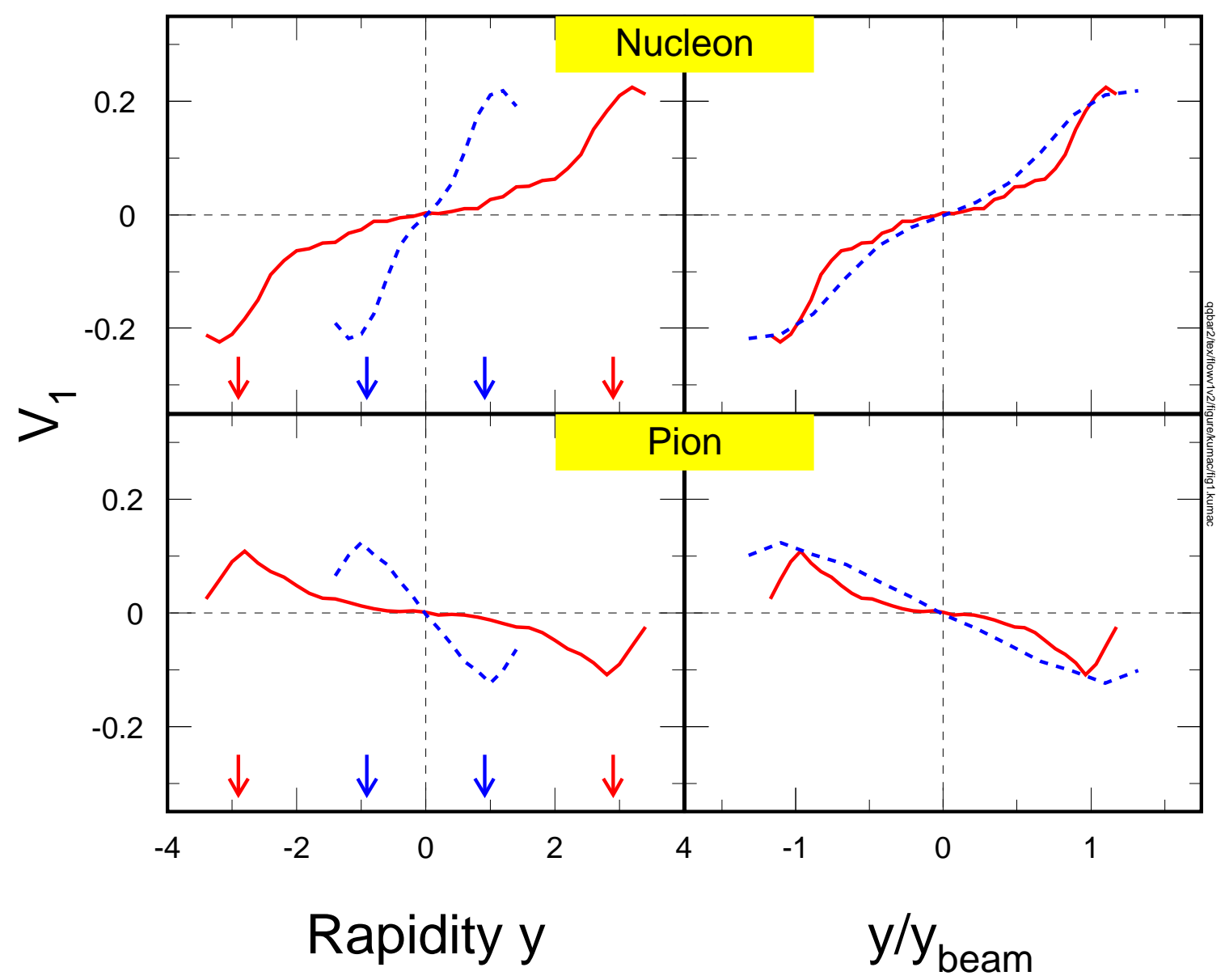

